\title{
WHY AND HOW ARE BUSINESS ECOSYSTEMS USING DIGITAL TECHNOLOGIES?
}

Camila Gonçalves Castro (camila.castro@ifmg.edu.br) - Federal Institute of Education, Science and Technology of Minas Gerais (IFMG).

Isabela Simões Zacharias (isabela.zacharias@usp.br) - University of São Paulo (USP).

Adriana Hofmann Trevisan (

Janaina Mascarenhas (jana.mascarenhas@usp.br) - University of São Paulo (USP).

\begin{abstract}
Digital technologies and information and communication technologies (ICT) are used in emergent business models. Previous research works show that the internet of things (IoT) and big data are the most common technology used in digital transitions. In the literature, it was found that ICT is fundamental to transition to industries 4.0. ICT is debated as an enabler to a green economy and productivity growth and collaborates with the business ecosystem development and innovation. Wherever studies do not tackle why and how these ecosystems use ICTs. This paper aims to disclosure the linking between these technologies to the main objective that they are used in different sectors of the economy, drawing a map of the usage and limitations. A systematic literature review was conducted to fill this gap, using the Scopus database and the keywords Business Models and digital technologies. The finds summarize the context of the use and presents a framework to show how the ecosystems embrace cooperation, consumer involvement, integration between partners on developments, and others. This paper contributes to elucidate the main use of technologies, helping new business models and research in this field, and defining efforts in future research.
\end{abstract}

Keywords: Business Ecosystems; Digital Technologies; systematic literature review; 


\section{INTRODUCTION}

The business ecosystem (BE) concept has evolved over the years. Moore (Moore, 1993) introduced the idea of BE inspired by its biological counterpart. According to him (Moore, 1993), the main purpose of pursuing a BE is to develop an innovative value that satisfies consumers through companies operating cooperatively and competitivity. BE concept is beyond a supply chain or an industrial ecology, but embraces a business perspective in which business context, including value capture, delivery, and creation is the central point (Tsujimoto et al., 2018). In this study, we used BE definition: "The ecosystem is defined by the alignment structure of the multilateral set of partners that need to interact in order for a focal value proposition to materialize" (Adner, 2016).

Several studies have addressed and pointed out mechanisms to BE development. One example is ecosystem coordination and collaboration that link ecosystem parts (Jacobides et al., 2018), including a supply chain structure until consumers (Tsujimoto et al., 2018) and actors network. Another example is ecosystem value creation and capture related to the complementarity among ecosystem parts (Jacobides et al., 2018) to either deliver a solution or connect distinct ecosystem partners using a platform (Pidun et al., 2019).

The employment of digital technologies (DTs) can assist BE in the mentioned challenges and corresponds to a new BE concept evolution. The digitalization concept has emerged as an exponential tool to allow new features and configurations that bring new market opportunities (Porter \& Heppelmann, 2014) through DTs usage. IoT (i.e., the connection between the physical and virtual system employing sensors) and Big Data (i.e., data management and analysis) (Nobre \& Tavares, 2017) are examples of DTs applied in BE (Hanelt et al., 2020). Digitalization opens up new forms of companies to create and deliver value to customers, sharing ideas and knowledge with partners without necessarily in contract-based coordination (Thomas \& Autio, 2020). So, Thomas and Autio (2020) affirm that digitalization can enable innovation and new business models, and the ICTs are being a structural change in society. DT can make market entry easier and expand the scope of firms (Gawer, 2020).

Even though digitalization has been associated with BE (Kuusisto, 2017) due to its potential to enhance BE performance, we still lack a systematic map of DTs usage in the BE context, to stimulate the discussion on how BE can use DTs across different sectors and purposes (Hanelt et al., 2020). Thus, this paper addresses the following fundamental research question:

Why and how are DTs being used in BE? 
Our main goal is to verify in real BE cases studies described in literature how and why DTs are affecting the BE. We investigated BE employing DTs such as IoT, Big Data, and Information and Communication Technology (ICT) from a Systematic Literature Review (SLR) approach. Our results provide both academic and practical perspectives, an initial discussion regarding the usage of DTs in BE in terms of why and how they are being employed.

\section{METODOLOGIA}

This study aims to comprehend the link between digital technologies (DTs) and the main objectives of why they are employed in business ecosystems (BE). To map this usage of DTs in ecosystems, we conducted a systematic literature review (SLR) targeting real BE cases. SLR followed (Tranfield et al., 2003) guidelines, and it was chosen because it allowed understanding the relationship based on an evidence-based approach. Table 1 shows the research protocol.

Table 1. Research Protocol

\begin{tabular}{ll}
\hline Scope & Details \\
\hline Purpose & Map the usage of DTs in business ecosystems \\
Database & Scopus \\
String & TITLE-ABS-KEY (("business ecosystem") AND ("Internet of things" OR \\
& "IoT" OR "Big Data" OR "Information communication* technology*" OR \\
& "ICT") \\
Time Frame & Until September 2020 \\
Unit of Analysis & Real business ecosystem case \\
Inclusion Criteria & Papers in English \\
& Papers published in journals or conferences \\
& Description of a business ecosystem case provided sufficient information to \\
& identify DTs and their usage \\
\hline
\end{tabular}

\subsection{Data Collection}

Scopus database was chosen because it includes a broader range of journals and conferences (Falagas et al., 2008) relevant to the topic. We formulated the string according to the main DTs described in the literature, i.e., the internet of things (IoT), Big Data, and information and communication technology (ICT) (Hakanen \& Rajala, 2018; Porter \& Heppelmann, 2014) associated with BE (Kuusisto, 2017). We search for real cases presented in the Scopus database until September 2020 that are (1) written in English, (2) published in journals or conferences, and (3) described in detail of a BE and DTs (innovation ecosystems and national ecosystem were excluded). 


\subsection{Data Analysis}

In total, 134 studies were found. We applied two filters, following the inclusion criteria. First, we evaluated the title, abstract, and article keywords. Thirty-eight were approved for the next filter. We analyzed the real cases using qualitative analysis (Miles et al., 2014) through content analysis (Elo \& Kyngäs, 2008). MAXQDA® software, which is a specific software for qualitative research, was employed during our investigation. The final sample is composed of 12 papers with 16 cases (Fig. 1).

We identified the purpose for which the technology was used in each case study, categorize, and present why the technologies are used in business ecosystems. Some cases gave us more than one technology or digital function within the ecosystem; therefore, the number of relationships between the functions of the technologies and their effect on the business ecosystem is larger than the number of cases presented.

In line with Kristoffersen et al. (2020), we classified the data flow that we found into three categories: collection, integration, and analysis, according to its data flow inside BE. We identified in these case studies applications where digital technologies were fundamental to the development of the business ecosystem.

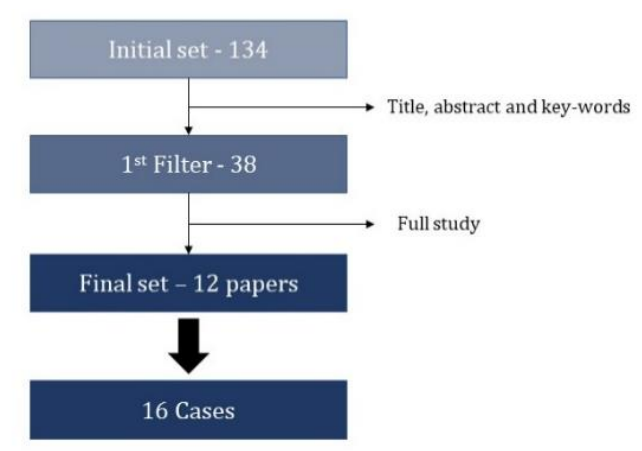

Fig. 1. Papers selection progress.

\section{RESULTS AND DISCUSSION}

We present in the following sections the results we achieved in this research. In section 3.1, we present the case studies addressed in our research. In section 3.2, we present the three whys digital technologies are used, and in section 3.3, we expose how this happens. Next, in section 3.4, we present the resulting framework with the map of use. 


\subsection{Cases Description}

We analyzed 16 real ecosystems cases employing DTs. The description and the ecosystem actors are presented in Table 2. The BE are from several areas such as health, parking, service provider, steel industry, home automation, mobility, farming, logistic, banking, and telecommunications. We stressed the ecosystem actors described to provide an overview of the essential partners that provide the ecosystems' value for each case.

Table 2. Details of Cases Description

Ecosystem Description
iMedBox is a health service based on equipment connected and controlled
by IoT. Patients possess iMedBox in their homes, which collect data from
them such as vital signals and medication activities and converted into
executable commands such as medication reminders
Parkeon is a parking ecosystem that utilizes IoT sensors (e.g. multi-space
meters) to offer several services to consumers. Services offering includes
making contactless payments and printing tickets
OpenStack is a collaborative ecosystem that aims to develop cloud
services. OpenStack is based on open technology development and meets
the needs of distinct (either public or private) stakeholders
MaterialCo is a steel industry ecosystem that monitors its products
throughout their life cycle. Their material (steel) is tracked using IoT to
collect data about the product and its usage by actors across the ecosystem
SmartCar is an ecosystem that offers data analysis to automobiles
independent of their brands. Smart Car employs IoT to provide a vehicle
on-board diagnostic
VR BE is an open platform to transform video processing into virtual
reality (VR). VR BE consumers can produce videos and content and boost
their consumer acquisition process

DIEM is a health-exercise ecosystem that aims to provide a smart environment for exercise. The ecosystem includes wearables, exercise

Ecosystem Actors

Ref.

Service providers (e.g., hospitals, pharmaceutical

companies, elderly homes), mobile device providers, content

providers (e.g. Google), medical device providers and insurance companies.

Parking manager (either public or private organization) and (Guittard et al., 2015) payment provider (such as banks and payment intermediates).

Private companies (e.g., Cisco, Dell, HP, IBM, Intel, among others) and public organizations (e.g., NASA, universities, and technologies centers).

MaterialCo and actors involved in the steel life cycle (e.g., primary production, maintenance, repair, assembly, and recycling companies).

$\mathrm{KT}$ (which is a telecommunication company that manage the (H. Yang et al., 2019) ecosystem) and Jastec (IoT sensors manufacturer company)

VR market involves SMEs companies (e.g., ThinkBridge and AlcaCruz) supplying large companies (e.g., LG Electronics)

Health club chain (i.e., offerings of exercises and personal training), manufactures of wearables devices (such as heart computers, personal training services, and gym equipment. DIEM is based rate monitors) and gym equipment, and companies that offer on interoperable smart gym technology employing a smart card to collect rehabilitation research and monitoring services. and analyze user data

The wellness ecosystem aims to offer services to promote quality of life to users. This ecosystem integrates wearables, apps, and activities plans

Mobile apps developers, wellness and recreation service companies, sensors manufacture and data analysis, and database companies.

Nest is a home automation ecosystem that consists of devices connected by IoT (thermostat). Through the device, it is possible to establish consumer preferences (e.g., heat systems) and reduce energy consumption. teams.

The user can control the thermostat by an app

AcquaCloud is an ecosystem focused on water refilling stations. This

Web administrators (such as Google and Explorer), shop ecosystem provides services to SMEs using a SaaS (software as a service) owners (e.g., water refilling stations), and consumers mobile app. AcquaCloud services include online reports of water transactions, inventory, and consumers' feedback

Celefish provides an $\mathrm{AI}$ and IoT ecosystem oriented to fish farming. There Celefish, financial organization, insurance companies, fish are two main services provided by Celefish. First, Celefish offers a digital farming, and fish farming suppliers platform to control fishing conditions such as water monitoring, oxygen controlling, and pest controlling. Second, this ecosystem produces data for financial institutions regarding fish farming lines of credit

SBI is a banking ecosystem that connects different banks aiming to enhance solutions offering and management. Banks are connected through the platform that provides data to be used on the decision-making process of loans and other financial applications

LoRa is a telecommunication ecosystem, which employs IoT for data exchange and connections. LoRa relies on partnerships to acquire consumers

SigFox is a telecommunication ecosystem that aims to collect data and exchange information through the ecosystem. Data is acquired using IoT devices that send these data to other companies (third parties' companies), which use those data to provide service and offerings

iCargo is a logistic ecosystem that relies on IoT to exchange information, monitor products and process to synchronize activities (e.g., warehousing, transport, management of pollution emission, carbon footprint)

Banks, consumers, and payment intermediates.

Teixeira et al., 2016)

(Hakanen \& Rajala, 2018)

(H. Yang et al., 2019)

(Annanperä et al., 2016)

(Annanperä et al., 2016)

(Makinen, 2014)

(Samonte et al., 2018)

(X. Yang et al., 2020)

Manufacturers (e.g., chip producers) and terminals.

(Kasai, 2019)

Devices partners (IoT devices, chips), data acquisition and data storage companies, application partners (analyses service providers).

3M (companies that want to boot its sustainable value proposition), DHL (virtual integrator), and Marlo (infrastructure provider)

LogiCon is a logistic ecosystem that objectives enhancement of efficiency Combi Terminal Twente (logistic operator), NexuZ (data and productivity. The ecosystem is based on a multi-sided platform that connects distinct user groups. In this case, the platform manages activities manager), and distinct user groups. 


\subsection{Why technology is used in the business ecosystem}

We identify three main categories of why DTs are used. The purposes target to (1) expand competitiveness, (2) create value and innovation, and (3) develop new business models each why is detailed in sequence.

\subsubsection{Expand the competitiveness of the ecosystem}

Technology is a facilitator for competitiveness. It permits improving the ecosystem without changing the business model or creating new solutions. The adoption of technologies facilitates exchanging and monitoring information, allowing the connection, and bringing together actors who would have previously been distant, hindering the improvement process of the ecosystem's performance.

BEs are using technologies to expand the scope of business and compete with ecosystems that already offered similar solutions to increase competitiveness. The case of OpenStack (Teixeira et al., 2016) is an example in which technology was developed to compete mainly with the services provided by Amazon Web Service in the sector of Big Data storage. In addition to the primary service offered, the use of technologies allowed improved solutions, since better communication was ensured, specifically with more efficient sharing of information.

The competitiveness of the ecosystem is also facilitated by connecting the actors for its development. DTs enable communication, data sharing, and information exchange at a higher speed. The use of IoT and BDA supports this connection. In iCargo and LogiCon (Boschian \& Paganelli, 2016) cases, these technologies allowed an alignment of logistics between ecosystem partners and facilitated orchestration activities.

The use of digital technologies to monitor and perform a predictive analysis allows the ecosystem to become more competitive by reducing activity risks. An example is that of Celefish (Xiaoping Yang et al., 2020), which guaranteed better productivity levels by monitoring the conditions of its fish farming, reducing the high risks associated with the activity. With the reports obtained, it was still possible to request financing since there was a more guarantee of return. 


\subsubsection{Value creation and innovation}

In this category, BE uses technologies to develop new solutions. BE can generate value and innovation in two ways: using DTs to improve their solution or offering the technology per si as the solution.

In the cases of Parkeon (Guittard et al., 2015) and Aquacloud (Samonte et al., 2018), IoT and Cloud were used as a tool for the process innovation of their solutions. In the Aquacloud case, the developer actors used the DT to offer new solutions to customers. Customers do not necessarily perceive the technologies involved, as in the Parkeon case, which developed parking monitoring services, informing consumers about the current conditions and providing services such as printing coupons.

Digital technologies have also enabled the creation of new products and services for endusers. Product innovation was the basis of the iMedbox (Pang et al., 2015), MaterialCo (Hakanen \& Rajala, 2018), SmartCar (H. Yang et al., 2019), and Nest (Makinen, 2014) cases. In these cases, end-users were the ones who used the technologies and had control over the operation, as in the case of Nest (Makinen, 2014), where customers installed thermostats in their homes and programmed them according to their needs. Another example is the SmartCar, where the sensor was installed inside the car to monitor its conditions.

\subsubsection{Business model development}

Finally, DTs can also be used to promote the creation of new partnerships and value that previously would not have been possible without ICT use. A mobile platform was created in the DIEM (Annanperä et al., 2016) case to connect the ecosystem actors to share information so that the participants could develop new business models. The technology was a facilitator for the actors to know each other better and seek new partnerships for developing business models.

The technologies can also be used for the expansion of consumers and markets. In the VR BE (H. Yang et al., 2019) case, the number of consumers using an ecosystem solution increased due to technology employment. The solution proposed with VR served for consumers to create their entertainment content on the platform, thus increasing and personalizing the content attracted new consumers. In the LoRa case (Kasai, 2019), the ecosystem implemented DTs to collect and exchange user information so that partners were able to expand and attract new customers for the solution. 


\subsection{How Technologies are used in business ecosystems}

According to Kristoffersen (2020) the data flow is a structural hierarchy in theory, not necessarily in practice, but represents how the data is processed and connected. This data flow process shows the potential of ecosystems to use DTs to improve productivity. As the use and domain are improved, so is the BE capability to understand the context and change the performance of the ecosystem's firms. This can represent resource coordination at a strategic level. The success of an ecosystem depends on a strategic alignment amongst the complementors.

The DTs used primarily to generate and collect data from different sources, about the product or the user were classified as Data Collection, as in (Pang et al., 2015), where the proposed device collected medial data about the user. The data collection is used to monitor product usage, identify users and products throughout their life cycle. We identified that IoT and Cloud were the leading technologies used in this category for the cases presented.

Table 3 Data Flow cases

\begin{tabular}{|c|c|c|c|}
\hline Data flow & $\begin{array}{l}\text { Function of } \\
\text { technology }\end{array}$ & Cases & Technologies \\
\hline \multirow[t]{4}{*}{ Collection } & Identify product & (Pang et al., 2015) & IoT \\
\hline & Identify user & (Pang et al., 2015) (A. Thomas, 2019) & IoT \\
\hline & Monitor the product & (H. Yang et al., 2019) (Boschian \& Paganelli, 2015; Kasai, 2019) & IoT \\
\hline & Storage & (Kasai, 2019; Samonte et al., 2018; Teixeira et al., 2016) & Cloud \\
\hline \multirow[t]{3}{*}{ Integration } & Automation & (X. Yang et al., 2020) & AI \\
\hline & Connect ecosystem & $\begin{array}{l}\text { (Annanperä et al., 2016; Boschian \& Paganelli, 2015; Kasai, 2019; H. } \\
\text { Yang et al., 2019) }\end{array}$ & $\begin{array}{l}\text { IoT, Mobile, } \\
\text { VR }\end{array}$ \\
\hline & $\begin{array}{l}\text { Information } \\
\text { exchange }\end{array}$ & $\begin{array}{l}\text { (Boschian \& Paganelli, 2015; Guittard et al., 2015; Kasai, 2019; A. } \\
\text { Thomas, 2019) }\end{array}$ & IoT, Mobile \\
\hline \multirow[t]{2}{*}{ Analysis } & Data analytics & (A. Thomas, 2019) & BDA \\
\hline & Predictive analytics & (Mäkinen et al., 2014; H. Yang et al., 2019) & BDA, IoT \\
\hline
\end{tabular}

Some of the BE studied use DTs to automate processes - like Celefish (Xiaoping Yang et al., 2020) that uses AI for fish farming, connect the ecosystem actors - like Wellness ecosystem (Annanperä et al., 2016) that use a mobile application to connect wearables that delivers value to customers, or to information exchange inside the ecosystem to so the actor can make decisions, like cases of Sigfox (Kasai, 2019) and LogiCon (Boschian \& Paganelli, 2016). We allocate these uses in the category Data Integration, where the data is contextualized, and the information collected is processed. The exchange of information is crucial to ecosystem survival. 
In the last category, data analysis, are the cases that use digital technologies to do a predictive analysis of the data, i.e., the technology is used to understand the data and automatically make decisions derived from it. We distinguish between data analytics and predictive analysis and identify in the cases the use of BDA and IoT. This type of appliance is seen in the case already cited Celefish and in the case of Nest (Makinen, 2014). In both, the technology is used to learn how the users behave and automatically change the product's behavior.

\subsection{Map of DTs usage in the BE context}

In Table 4 we present the map of why and how business ecosystems are using digital technologies. The map is an overview of the relationship between the data flow and why the DT was used, providing some insights and highlighting potentials practical developments.

Shipilov and Gawer (2020) theorizes about the importance of complementarities and the interdependence of the ecosystem's organizations to achieve success. According to them, the interdependence can be created or enhanced by technological development, as we see in our case studies. The crossing between connecting actors, ecosystems, and information exchange, in table 3 , is the point where we perceive the most significant number of cases, in line with Shipilov \& Gawer (2020) . This means that the integration of data helps expand the ecosystems while facilitates the organization of complementary resources.

Table 2 Map of digital technology usage in business ecosytems context

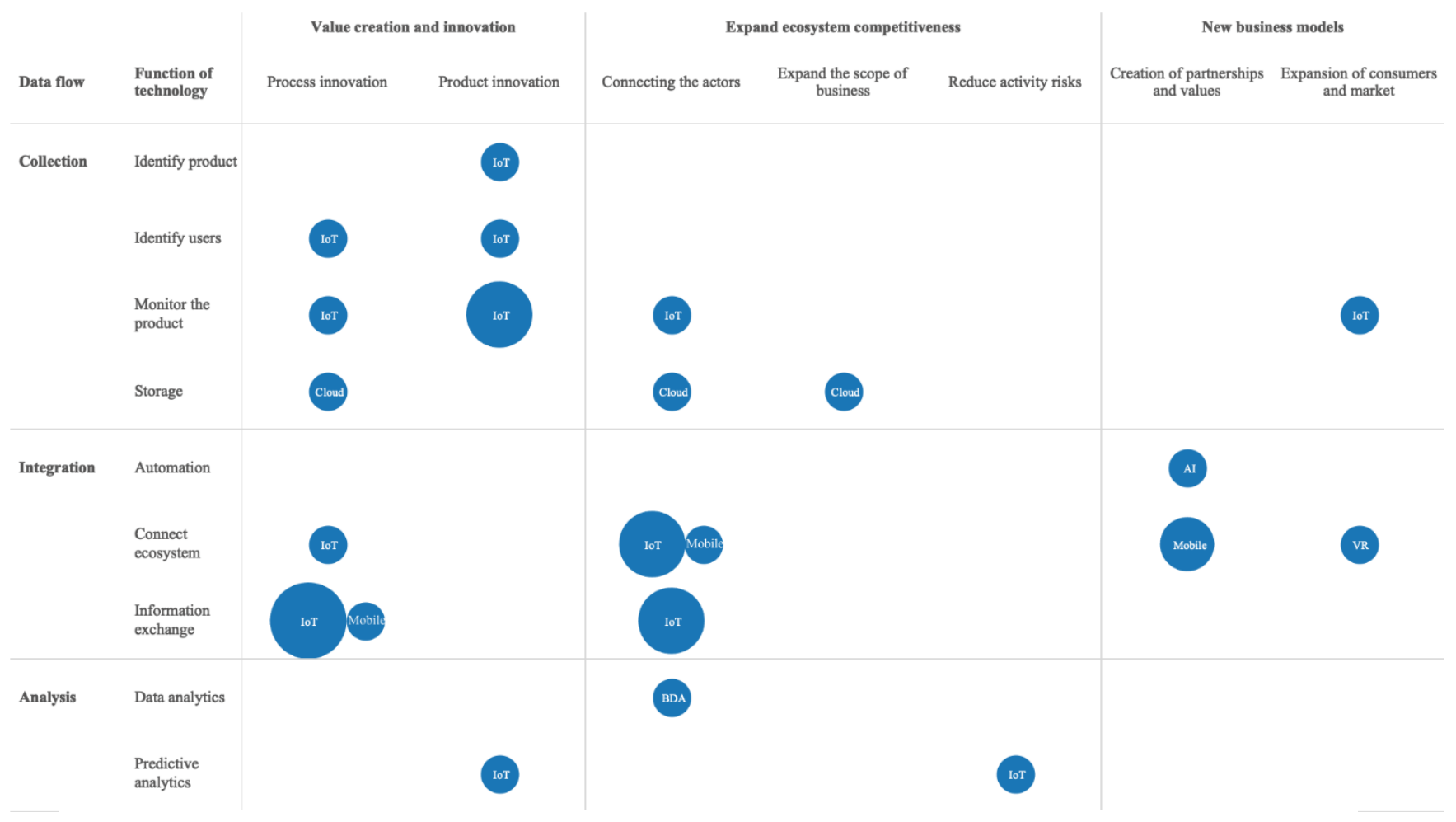

Note: The circles sizes show 1,2 or 3 cases 
The DTs are an enabler of the formation, expansion, and consolidation of the BE. Some of the business models seen in the cases are only possible with digital technologies as a supporter. DTs have great relevance in the business ecosystem's life cycle, transitioning to high maturity levels of development and management. This means that an isolated firm can not develop the ecosystem, this has to be coordinated action between loosely connected actors, so the DTs are a useful mechanism to facilitate that.

The population of cases decreases as more resources and infrastructure for data analysis are needed, in this study, only 3 cases were in data analysis. It is noticed that the beginning of the development in data use starts in data collection and in the value proposition. At the point where the ecosystem dominates this value proposition, the data flow moves to data integration, and we can see the expansion of BE. To allow new business models to rise integrated with the data flow, we need to understand how to transform these data flows into new value propositions for the business ecosystem as a whole, not isolated actions by firms.

The concentration of cases in the cross of data collection, integration, and value creation is evidence of DTs uses in BE - and studies about, are rising now. We believe that these topics' maturity will probably involve the creation of new business models with data analytics, an empty space in our map.

Some of the challenges that BE has to address soon are the data's safety and reliability of the data flow inside the ecosystem as the firms do information exchange. Another challenge is to create business models that can monetize new developments with digital technologies, not necessarily through product creation.

\section{CONCLUSIONS}

In this paper, we attempt to answer the question 'Why and how are DTs being used in BE?'. To address that question, we systematically examined empirical cases from the literature, providing future researchers a grounded basis on ecosystems using digital technologies and making two main contributions from the framework we developed.

First, in this framework, the relation of Why and How categories are essential to visualize how ecosystems have used digital technologies, contributing to the field's clarification and helps researchers find new avenues to investigate digital technologies' role in business ecosystems.

Second, from the framework, we can raise further discussion and have insights for developing strategies for the future uses of digital technologies. The bridge between BE and 
DTs is urgent when we are moving to a data-based culture. Thus, the ecosystem's leaders can be more assertive in the search for solutions and directions.

In this study, we recognized patterns of DT use and opened a few points to theoretical and empirical research. It is necessary to address the BE's maturity in future research and investigate DTs' roles in this process. Another important research topic is how DT integrates into capabilities to innovate in ecosystems as we move to a data-driven culture.

In some cases (H. Yang et al., 2019; X. Yang et al., 2020), the owner or developer of the DT was not the orchestrator of the ecosystem; it participates as a complement to deliver the value that the ecosystem provides. That opens an opportunity for future research about the actors' participation in a business ecosystem that is highly dependent on DT.

We believe that this study is one step towards a broader comprehension of the integration of DTs in the BE. The cases analyzed demonstrates that there are still many possibilities and ways to use the technologies in data-driven BE.

\section{ACKNOWLEDGEMENTS}

This work was supported by the Higher Education Personnel Improvement Coordination (Capes) - Finance Code 001 .

We acknowledge the support of Instituto Federal de Minas Gerais - Campus Congonhas to our work.

\section{REFERENCES}

Adner, R. (2016). Ecosystem as Structure: An Actionable Construct for Strategy. Journal of Management, 43(1), 39-58. https://doi.org/10.1177/0149206316678451

Annanperä, E., Liukkunen, K., \& Markkula, J. (2016). Managing emerging business ecosystems - A knowledge management viewpoint. AMCIS 2016: Surfing the IT Innovation Wave - 22nd Americas Conference on Information Systems.

Boschian, V., \& Paganelli, P. (2016). Business models for advanced ICT in logistics. In Contributions to Management Science. https://doi.org/10.1007/978-3-319-17419-8_2

Elo, S., \& Kyngäs, H. (2008). The qualitative content analysis process. Journal of Advanced Nursing, 62(1), 107-115. https://doi.org/10.1111/j.1365-2648.2007.04569.x

Falagas, M. E., Pitsouni, E. I., Malietzis, G. A., \& Pappas, G. (2008). Comparison of PubMed, Scopus, Web of Science, and Google Scholar: strengths and weaknesses. The FASEB Journal, 22(2), 338-342. https://doi.org/10.1096/fj.07-94921sf

Gawer, A. (2020). Digital platforms' boundaries: The interplay of firm scope, platform sides, and digital 
interfaces. Long Range Planning, October 2018, 102045. https://doi.org/10.1016/j.lrp.2020.102045

Guittard, C., Schenk, E., \& Burger-Helmchen, T. (2015). Crowdsourcing and the evolution of a business ecosystem. In Advances in Crowdsourcing. https://doi.org/10.1007/978-3-319-18341-1_4

Hakanen, E., \& Rajala, R. (2018). Material intelligence as a driver for value creation in IoT-enabled business ecosystems. Journal of Business and Industrial Marketing, 33(6), 857-867. https://doi.org/10.1108/JBIM11-2015-0217

Hanelt, A., Bohnsack, R., Marz, D., \& Antunes Marante, C. (2020). A Systematic Review of the Literature on Digital Transformation: Insights and Implications for Strategy and Organizational Change. Journal of Management Studies. https://doi.org/10.1111/joms.12639

Jacobides, M. G., Cennamo, C., \& Gawer, A. (2018). Towards a theory of ecosystems. Strategic Management Journal, 39(8), 2255-2276. https://doi.org/10.1002/smj.2904

Kasai, E. (2019). LPWA alliance strategy: Example of sigfox alliance in Japan. PICMET 2019 - Portland International Conference on Management of Engineering and Technology: Technology Management in the World of Intelligent Systems, Proceedings. https://doi.org/10.23919/PICMET.2019.8893921

Kristoffersen, E., Blomsma, F., Mikalef, P., \& Li, J. (2020). The smart circular economy: A digital-enabled circular strategies framework for manufacturing companies. Journal of Business Research, 120(August), 241-261. https://doi.org/10.1016/j.jbusres.2020.07.044

Kuusisto, M. (2017). Organizational effects of digitalization: A literature review. International Journal of Organization Theory and Behavior, 20(3), 341-362.

Makinen, S. J. (2014). Internet-of-things disrupting business ecosystems: A case in home automation. IEEE International Conference on Industrial Engineering and Engineering Management, 2015-Janua, 14671470. https://doi.org/10.1109/IEEM.2014.7058882

Miles, M. B., Huberman, A. M., \& Saldaña, J. (2014). Qualitative Data Analysis. A Methods Sourcebook (A. S. University (ed.); 3 edition). SAGE.

Moore, J. F. (1993). Predators and prey: A New Ecology of Competition. Harvard Business Review, 71(3), 7586.

Nobre, G. C., \& Tavares, E. (2017). Scientific literature analysis on big data and internet of things applications on circular economy: a bibliometric study. Scientometrics, 111(1), 463-492. https://doi.org/10.1007/s11192-017-2281-6

Pang, Z., Zheng, L., Tian, J., Kao-Walter, S., Dubrova, E., \& Chen, Q. (2015). Design of a terminal solution for integration of in-home health care devices and services towards the Internet-of-Things. Enterprise Information Systems, 9(1), 86-116. https://doi.org/10.1080/17517575.2013.776118

Pidun, U., Reeves, M., \& Schüssler, M. (2019). Do you need a business ecosystem? In BCG Henderson Institute.

Porter, M. E., \& Heppelmann, J. E. (2014). How Smart, Connected Products Are Transforming Competition. Harvard Business Review, 92(11), 64. 
Samonte, M. J. C., Javier, J. P. R., Mataga, L. M., \& Timbang, T. T. (2018). AquaCloud: A SaaS disruptive innovation for enterprise business ecosystem. ACM International Conference Proceeding Series, 84-89. https://doi.org/10.1145/3230348.3230353

Shipilov, A., \& Gawer, A. (2020). Integrating Research on Inter-Organizational Networks and Ecosystems. Academy of Management Annals, 14(1). https://doi.org/https://doi.org/10.5465/annals.2018.0121

Teixeira, J. A., Mian, S. Q., \& Hytti, U. (2016). Cooperation among competitors in the open-source arena: The case of OpenStack. 2016 International Conference on Information Systems, ICIS 2016.

Thomas, L. D. W., \& Autio, E. (2020). Innovation Ecosystems in Management: An Organizing Typology. Oxford Research Encyclopedia of Business and Management, July. https://doi.org/10.1093/acrefore/9780190224851.013.203

Tranfield, D., Denyer, D., \& Smart, P. (2003). Towards a methodology for developing evidence-informed management knowledge by means of systematic review. British Journal of Management, 14, $207-222$. https://doi.org/10.2307/249689

Tsujimoto, M., Kajikawa, Y., Tomita, J., \& Matsumoto, Y. (2018). A review of the ecosystem concept Towards coherent ecosystem design. Technological Forecasting and Social Change, 136, 49-58. https://doi.org/10.1016/j.techfore.2017.06.032

Yang, H., Kim, S. Y., \& Yim, S. (2019). A case study of the Korean government's preparation for the fourth industrial revolution: Public program to support business model innovation. Journal of Open Innovation: Technology, Market, and Complexity, 5(2). https://doi.org/10.3390/JOITMC5020035

Yang, X., Cao, D., Chen, J., Xiao, Z., \& Daowd, A. (2020). AI and IoT-based collaborative business ecosystem: A case in Chinese fish farming industry. International Journal of Technology Management, 82(2), 151171. https://doi.org/10.1504/IJTM.2020.107856

Yang, Xiaoping, Cao, D., Chen, J., Xiao, Z., \& Daowd, A. (2020). AI and IoT-based collaborative business ecosystem: a case in Chinese fish farming industry. International Journal of Technology Management, 82(2), 151-171. https://doi.org/10.1504/ijtm.2020.10030058 